\title{
Den öffentlichen Raum „hacken“ und die Menschen beeinflussen
}

\author{
Irwan Ahmett \\ Der dazugehörige Video-Beitrag und eine englischsprachige Version des Textes sind im HTML-Format \\ auf der Seite der Zeitschrift zu finden.
}

The english version of the text as well as the video can be found in the HTML-version on our homepage. An english abstract can be found at the end of the article.

1942 versprach Japan in seiner Funktion als der „ältere asiatische Bruder" Indonesiens das Land nach dreieinhalb Jahrhunderten von der niederländischen Kolonisierung zu befreien. Stattdessen wurden die Japaner_innen aber während der nächsten dreieinhalb Jahre selbst zu Kolonisator_innen. Zum Zeitpunkt als die Atombomben auf Hiroshima und Nagasaki fielen und Japan den Krieg verlor, war Indonesien immer noch eine Kolonie. Am 17. August 1945 riefen Freiheitskämpfer dann die unabhängige Republik Indonesien aus. Doch die Alliierten - die Briten, die US-Amerikaner und natürlich auch die Niederländer zeigten sich unwillig, die Kolonie ziehen zu lassen. Einen Monat nach der Unabhängigkeitserklärung durch Sukarno und Hatta (Indonesiens ersten Präsidenten bzw. Vizepräsidenten) kehrten die Niederländer zurück und hissten eigenmächtig auf großen Gebäuden ihre Nationalflagge. Dieser provokative Akt ließ die Lage eskalieren. Im Yamamoto-Hotel in Surabaya auf Ost-Java kam es zu einem folgenreichen Vorfall: Die Indonesier zwangen die Niederländer, ihre rot-weiß-blaue Trikolore einzuholen, was in einer blutigen Schlacht mündete. Hinter diesem Ereignis vom 10. November 1945 verbergen sich jenseits des offenen Krieges zwischen Indonesien, den Niederlanden und den Alliierten eine Reihe von Geschichten. Die Geburt der indonesischen Nation fand zu einem Zeitpunkt statt, als die Welt in einer tief greifenden Krise steckte, die ihr Aussehen für immer verändern sollte.

Es ist das Zerreißen der Flagge, das ich an diesem Vorfall bedeutungsvoll finde. Eine Gruppe mutiger junger Männer war, mit erbeuteten Waffen und Bambusspeeren ausgerüstet, auf den Fahnenmast geklettert, auf dem die niederländische Trikolore aufgezogen war. Es war ein ungleicher Kampf, da die Alliierten überlegene Waffen hatten, doch die wirkliche Macht war auf der Seite dieser jungen Männer, die bereit waren, ihr Leben zu lassen. 
Schlißlich rissen sie den blauen Streifen der niederländischen Fahne ab, sodass nur die indonesischen Nationalfarben rot und weiß übrigblieben. Das Bild dieses heroischen Akts, das die Medien verbreiteten, löste im ganzen Land Widerstandsbewegungen aus. Für mich ist dieser Vorfall die erste Intervention im öffentlichen Raum Indonesiens und damit die wichtigste Inspirationsquelle für meine eigenen Interventionsversuche und Kunstprojekte.

65 Jahre nach der Unabhängigkeitserklärung Indonesiens kletterte ein Mann mittleren Alters in Jakarta verbotenerweise auf das Dach des indonesischen Parlamentsgebäudes. Seine Botschaft waren drei Wörter, die sehr gut die Stimmung im Land zum Ausdruck brachten. Seine „Waffe“ war eine Farbspraydose für zwei US-Dollar. Pong Harjatmos Aktion stieß auf öffentliche Resonanz, weil klar war, dass es nicht mehr ausreicht, sich von der Politik abzuwenden und diesem ruhmreichen Gebäude lediglich den Mittelfinger zu zeigen. Die zahllosen Korruptionsaffären sind nur die Spitze eines Eisbergs. Harjatmo, ein einfacher Mann, überbrachte den Volksvertreter_innen eine Botschaft, die aus der Tiefe seines Gewissens kam: „Honest, Fair, Firm“.

Die eigenen Gedanken in Handeln zu überführen ist entscheidend, wenn wir unsere Ziele erreichen wollen. In bestimmten Situationen ermöglicht dein Verstand deinem Körper, dich mit bestimmten Mitteln zu verteidigen oder andere anzugreifen. In vielen Fällen ist die Waffe ganz einfach und besteht aus frei zugänglichem Material. So benutzten die indonesischen Freiheitskämpfer Speere aus Bambus, das in ihren Gärten wuchs. Harjatmo hatte seine Spraydose im Baumarkt gekauft.

Waffen und Kämpfe sind untrennbar. Ich war immer überzeugt davon, dass die Effizienz einer Waffe vor allem davon abhängt, wie sie eingesetzt wird und nicht von der Kraft der sie nutzenden Person.

- In der javanesischen Version des Mahabharata gibt es die Chronik der fünf Pandava-Ritter, die gegen das Königreich ihrer Verwandten, der Kaurava-Brüder, kämpften. Diese epische Schlacht, die als Bharatayudha bezeichnet wird, ist eine Schlacht zwischen zwei Supermächten. Der Höhepunkt der Geschichte ist, wenn Karna, ein Kaurava-Ritter, dem Riesen Gatotkaca gegenübertritt, einem PandavaRitter, der fliegen und Feinde von der Luft aus angreifen kann, so wie ein Adler eine Maus reißt. Gatotkacas Haut ist unverwundbar, er hat „Muskeln aus Draht und Knochen aus Stahl“. Karna jedoch hat eine mächtige Waffe, die er sein ganzes Leben lang aufbewahrt hat: den Konta-Pfeil, den man nur einmal verwenden kann. Er schießt den Pfeil ab, der zum ersten und letzten Mal trifft und sein Opfer fordert.

- Eine Waffe kann gegen ihren Besitzer gewendet werden. Es war einmal ein hindubuddhistisches Königreich auf Java, in dem ein Rebell namens Ken Arok einen Kris (einen Dolch mit wellenförmiger Klinge) besaß, der von dem berühmten Schmied Mpu Gandring angefertigt worden und mit einem Fluch beladen war. Ken Arok vernichtete das Königreich Singhasari auf Ost-Java. Sein Kris war in viele politische Intrigen verwickelt und an der Ermordung der Eliten der SinghasariDynastie beteiligt. Am Ende richtete er sich gegen seinen Besitzer und tötete ihn und seine Nachfahren bis in die siebte Generation hinein. 
- Heutzutage hat die Jugend immer eine „Waffe“ zur Hand - das Mobiltelefon. Wo sie auch sind, sie sind stets verbunden und in der Lage, mit einfachen Fingerbewegungen die ganze Welt an ihren Emotionen teilhaben zu lassen.

Ich möchte hier zwei meiner wichtigsten Waffen, mit denen ich im öffentlichen Raum interveniere, vorstellen. Bei der ersten Waffe handelt es sich ums „Spielen“. Es ist eine natürliche Fähigkeit/Aktivität, die wir aber oftmals im Laufe des Erwachsenwerdens verlernt haben. Spielen erweitert unseren Verstand und verleiht uns eine unendliche Kreativität. Vielleicht habe ich als Kind zu viele Jackie-Chan-Filme gesehen, aber ich ziehe Freihandstrategien als Waffe vor und konzentriere mich allein auf die Vorstellung, den „Feind“ zu besiegen. Zu diesen Strategien gehört es, Fundstücke neu zu interpretieren, Gewohnheiten durcheinander zu bringen, sich über Gehorsam lustig zu machen, eine spezifische Situation zu kreieren, an einem ganz bestimmten Schauplatz die Gegebenheiten zu verändern und andere Formen der Guerillataktik gegen ein übermächtiges System einzusetzen. Die zweite Waffe ist etwas Atmosphärisches, etwas Unsichtbares, das aber reale Auswirkungen hat - „wirksame Distribution“, online oder im realen Leben.

Diese beiden Waffen reichen jedoch nicht aus, solange sie nicht von einer adäquaten Taktik begleitet werden. Mit Taktik verbindet man oft etwas Grobes und Anarchisches, etwas, mit dem sich jedes Mittel rechtfertigen lässt. Für mich geht es bei Taktik allerdings um die Frage, wie ich mit meiner Waffe das richtige Ziel treffen kann.

Hier ein Beispiel für eine solche Taktik: Gehe zu deinem Haus, zünde es an und mache dich davon! Ich bin mir sicher, dass ich dazu nicht den Mut hätte, aber in Indonesien haben Menschen in der Vergangenheit genau dies getan. Vier Monate nach dem 10. November 1945 beschlossen rund 200.00o Menschen in Bandung (West-Java), ihre Stadt niederzubrennen, um zu verhindern, dass sie wieder vom Westen kolonisiert wird. Diese Aktion ging als das „Flammenmeer von Bandung“ (Bandung Sea of Fire) in die Geschichte ein. Das löste einen regelrechten power boost aus, ähnlich wie im Fall von Gatotkatca: Als der von dem Konta-Pfeil - Karnas tödlicher Waffe - im Flug getroffen wird, weiß er, dass er bald sterben wird. Noch beim Aufprall gelingt es ihm, Tausende seiner Feinde mit seinem Riesenkörper, den er zuvor noch vergrößert hat, zu zerstören. Wenn wir im Leben nichts mehr zu verlieren haben, haben wir die Freiheit, alles zu tun!

Während des indonesischen Unabhängigkeitskriegs trugen auch viele der Frauen in Bandung, die für ihre Schönheit berühmt sind, Waffen und beteiligten sich am Kampf. Als ich im Internet nach dem (historischen) „Flammenmeer von Bandung“ suchte, war ich überrascht, wie viele Teenager-Liebesgeschichten und Berichte über Sexaffären unter diesem Stichwort zu finden waren. In Indonesien - dem größten muslimischen Land der Welt - sind bestimmte Aspekte des Unabhängigkeitkampfes des Landes zu einem Streitpunkt geworden. Im Jahr 2001 kam es zu einem Skandal: Zum ersten Mal verbreitete sich ein Sexvideo eines Paares aus Bandung in Windeseile in der Öffentlichkeit. Diese Amateuraufnahme trug den Titel „Bandung Sea of Fire of Romance“. Daraufhin folgten viele weitere skandalträchtige Sexvideos. Anscheinend gab es einen dramatischen 
Bedeutungswandel: weg vom heroischen Kampf hin zum „Kampf zweier Liebenden im Bett. Ja, so ist die Technik: Sie verbreitet sich mit Lichtgeschwindigkeit und hat zwei gegensätzliche Seiten.

Im Folgenden beschreibe ich die sechs Taktiken, die bei meinen urbanen Interventionen zur Anwendung kommen:

\section{Ich arbeite nicht kommerziell}

Es ist nicht einfach, als Künstler in einem Land zu überleben, dessen kulturelle Infrastruktur nicht besonders entwickelt ist und wo ein sehr konservativer Kunstgeschmack vorherrscht. Ich hatte das Angebot, sowohl für eine berühmte globale Schuhmarke als auch für die größte Internet-Suchmaschine in der Welt zu arbeiten und mit einem Kunst- und Interventionsprojekt deren Produkte in Jakarta zu bewerben. Ich habe das als ein großes Dilemma erlebt, aber zuletzt lehnte ich diese Aufträge ab. Ich habe eine Ausbildung als Grafikdesigner, daher verstehe ich nur zu gut, wie Werbung funktioniert und wie sie der Öffentlichkeit etwas vormacht. Wer auch immer diese Traumwelten produziert - die Menschen sind dafür offen und werden ihnen ihre Lügen abkaufen. Darüber hinaus sind die Länder der Dritten Welt immer schon lukrative Märkte für die Länder der Ersten Welt gewesen.

Um zu überleben, ziehe ich es vor, mit Menschen zusammenzuarbeiten, die ein ähnliches Unbehagen umtreibt, und gehe Tauschgeschäfte ein, bei denen Geld keine Rolle spielt. Mir ist es wichtig, die Menschen zu unterstützen, die mir am nächsten sind, und Vertrauen aufzubauen. Diese Taktik erlaubt es mir, in meiner künstlerischen Arbeit frei zu sein, weil es keine weiteren Absichten und Hintergedanken gibt. Indirekt ist damit auch verbunden, dass ich mir ein stabiles Netzwerk aufbauen musste, das tragfähiger ist als die bestehende offizielle Infrastruktur.

Bei meinem Projekt TV Milisi, an dem sich eine Gruppe von hier nicht näher benannten Menschen beteiligte, ging es darum, mithilfe von Universalfernbedienungen die Kontrolle über die Wahl der Fernsehkanäle im privaten und öffentlichen Raum zu erlangen. Wir wechselten auf andere Sender oder schalteten Fernseher aus. Ich machte das Fernsehen zum Gegenstand einer Aktion, da die meisten Sender nur an den Zuschauerzahlen orientiert sind, keinerlei Wert auf Qualität legen und geistige Trägheit fördern. Fernsehen ist eine Sucht: Die Menschen sitzen wegen der materialistischen Traumwelten, die sie dort zu sehen bekommen - unentwegt vor der Glotze, als ob sie über das Fernsehen die wahren sozialen und gesellschaftlichen Probleme vergessen könnten.

\section{Dynamik erzeugen}

Sondang Hutagalung, ein Jurastudent kurz vor dem Abschluss und Menschenrechtsaktivist aus Jakarta, war keine 22 Jahre alt, als er sich im Jahr 2011 das Leben nahm. Er verbrannte sich vor dem Präsidentenpalast in Jakarta. Der Grund war vermutlich seine herbe Enttäuschung angesichts der schwierigen Lage der Menschenrechte und mangelnder Rechtstaatlichkeit in Indonesien. Die Reaktionen auf seine Tat fielen ganz anders aus als 
bei der Selbsttötung eines Obstverkäufers in Tunesien, die zum Rücktritt von Präsident Zine El Abidine Ben Ali geführt hatte. Die habgierigen Machthaber in Indonesien ließen sich von Sondangs Opfer so gut wie gar nicht beeindrucken. Ich vermutete, dass im indonesischen Fall die notwendige Dynamik gefehlt hat. Aber kann man so eine Dynamik einfach nach dem Schneeballprinzip entfachen? Um das herauszufinden, machte ich eine Aktion auf einer illegalen Müllhalde. Alle wissen, dass es falsch ist, Müll einfach irgendwo abzuladen, aber die Leute lassen sich nicht davon abbringen. In Indonesien gibt es keinen Schnee, aber wir haben jede Menge Müll. Ich beschloss also, Müll Stück für Stück aufzulesen und zu einem Ball zu formen. Anfangs war ich ganz allein und auch etwas frustriert. Ich hatte Mühe, die Umstehenden dazu zu bringen, sich an meiner Aktion zu beteiligen. Sie dachten wohl, ich sei verrückt. Als der Ball aber eine gewisse Größe erreicht hatte, begann die Interaktion und ein neues Spiel zwischen mir und den Menschen um mich herum. Vor allem die Kinder halfen mir dabei, den Müllball immer größer werden zu lassen. Sie wirkten dabei sehr glücklich. Ich war sehr gerührt, als sich am Ende beinahe alle Umstehenden aktiv an der Aktion beteiligten. Mittels Crowdfunding kam für die Aktion Trashball eine finanzielle Unterstützung zustande, sodass der Ball ein Jahr später mehr als 300 Kilogramm wog. Ein solches Spiel kann zum Auslöser für eine kollektive Erfahrung und für Gespräche über bestimmte Probleme werden. Spielen kann uns dazu bringen, Probleme anders wahrzunehmen.

\section{Die Emotionen der Öffentlichkeit für sich gewinnen}

An einem Nachmittag im August 2011 ging ich durch die Straßen in London. Es war ungewöhnlich ruhig. Dann hörte ich die Martinshörner von Feuerwehrwagen und blickte in die angespannten Gesichter von Polizisten auf Pferden. Ein paar Stunden später wurde mir klar, dass die Stadt wie gelähmt war. Unruhen und Plünderungen hatten zu einer chaotischen, kriegsähnlichen Atmosphäre geführt. Ich hatte das schon einmal erlebt, und zwar während der viel schlimmeren Aufstände in Jakarta im Jahr 1998, die noch viel tragischer endeten, weil es dabei auch zu rassistischen Angriffen und zu Vergewaltigungen kam. Damals war die multikulturelle Grundlage des Landes bedroht. Dieser Aufstand fand am Ende der Suharto-Diktatur statt. Ich konnte dagegen nicht verstehen, wie es in London zu einem Aufstand hatte kommen können. Ich war irritiert, als sich die Unruhen nach Liverpool, Birmingham, Nottingham und in andere Städte ausbreiteten. Später konnte die Lage wieder unter Kontrolle gebracht werden, aber das Trauma sollte nicht so leicht verschwinden. Die Stimmung in London blieb angespannt, überall patrouillierte die Polizei, und selbst das sonnige Wetter vermochte es nicht, die Londoner zum Lächeln zu bringen. Aber mithilfe eines Spiels konnten wir die Atmosphäre verändern. Vor der Intervention fühlte man gar nichts, aber danach fühlte man sich glücklich. Oder man dachte gerade an gar nichts, und dann kam einem etwas in den Sinn. Bei dem Projekt Squeaky Sidewalk produzierte ich spielerisch(e) Geräusche in der superhektischen Stadt London, indem ich auf den Bürgersteigen an verschiedenen Stellen quietschendes Spielzeug unter den gelockerten und beschädigten Gehwegplatten versteckte.[1] 


\section{4. Öffentlichen Raum beanspruchen und besetzen}

Im Jahr 2011 hatte ich erstmalig Gelegenheit, für drei Monate in den Niederlanden zu leben. Ich interessiere mich dafür, welche Beziehung die Menschen in Europa zum öffentlichen Raum haben. In Jakarta ist der öffentliche Raum ungastlich geworden aufgrund der Bedrohung durch Kriminalität, ständiger Verkehrsstaus, Umweltverschmutzung und der Aneignung des Raums für persönliche Zwecke. So kann es vorkommen, dass die Anwohner_innen einfach eine Straße sperren und sie zu einem temporären Fußballplatz, dem Schauplatz einer religiösen Zeremonie oder Hochzeitsfeier oder auch nur zu einem Ort für das Trocknen von Wäsche machen. Der Staat und die Behörden haben nicht genügend Macht um alles zu kontrollieren. Genau wie Ken Arok sind sie allzu sehr damit beschäftigt, ihre vorhandene Macht zu bewahren. Ich war sehr beeindruckt, wie ordentlich es in Europa zugeht, aber nach einem Monat langweilte ich mich. Es gibt viele große öffentliche Plätze, aber alles ist dort stark reglementiert. Das System hat alles unter Kontrolle - oder ist das nur die Perspektive von jemanden wie mir, der an das Leben in einer chaotischen Stadt gewöhnt ist? Der Gedanke beunruhigt mich, dass bei einer Neugestaltung öffentlicher Plätze in Jakarta es die großen Unternehmen sein werden, die vor allem davon profitieren. Dann ist es mir lieber, wenn meine Stadt so bleibt, wie sie jetzt ist.

Normalerweise wird der öffentliche Raum von zwei Gruppen beherrscht: dem Kapital und dem Staat. Dem Kapital geht es selbstverständlich darum, soviel Gewinn wie möglich zu erzielen. Der Staat will das bestehende System aufrechterhalten. Ich schlug meinen neuen Freunden in den Niederlanden neue Methoden vor, wie man den öffentlichen Raum beanspruchen und für sich vereinnahmen kann, indem man auf bestimmte Situationen mit spontanen kollektiven Aktionen antwortet. Es beeindruckt mich, wie Mikroorganismen in den menschlichen Körper eindringen, wenn das Immunsystem eines Menschen geschwächt ist. Sie bringen den Körper durch ihre Intervention dazu, in anderer Weise als sonst zu reagieren. Wir griffen diese Idee in der Aktion Organism (Luchtalarm)[2] auf, bei der wir auf die einmal im Monat überall in Holland stattfindenden Probealarme reagierten, bei denen das Katastrophenwarnsystem getestet wird.

\section{Ein System schaffen und infiltrieren}

Es mag naiv klingen, aber ich bin es wirklich nicht gewohnt, in einem Land voller Videoüberwachungskameras zu leben. Mir fiel dies in den USA auf. Angst ist ein natürliches menschliches Gefühl, und man kann eine Gesellschaft leicht über ein wirkungsvolles Management der Angst kontrollieren. Mir kam es so vor, als ob uns die ganze Zeit über ein Geist beobachten würde und als ob sein ,Auge‘ die Kraft hätte, uns alle zu domestizieren.

Als ich dort lebte, setzte ich mehrere Projekte um. Eines hieß Light Spam. Ich verwendete einen Spiegel und lenkte das Sonnenlicht auf die Überwachungskameras um, um Nachrichten im Morsealphabet zu versenden. Bei einer anderen Aktion, Emergency Toilet, spielte ich mit den Überwachungskameras Verstecken. Es war nicht einfach, in den USA 
eine öffentliche Toilette zu finden - woraufhin ich Verkehrsanlagen zu Notfalltoiletten umfunktionierte.

\section{Eine Verschwörung anzetteln}

Im Jahr 2012 führte ich ein Projekt namens Very Very Important Fish durch.

Jakarta hat fast immer während der Regenzeit mit Überschwemmungen zu kämpfen. Das Problem besteht seit Jahren, und weder die Regierung noch die Bevölkerung unternehmen wirklich etwas dagegen. Der Müll türmt sich auf und verstopft die Schleusen; zugleich ist das Bewässerungssystem sehr schlecht, da die Regierung kurzfristigen populistischen Maßnahmen den Vorzug vor effektiveren langfristigen Lösungen gibt. Noch viel bedauerlicher ist es, dass die Menschen Müll in den Fluss werfen, der wie der Schlund des Todes aussieht. Die Fische werden krank und sterben, weil das Wasser so stark verschmutzt ist. In manchen Gebieten der Stadt müssen die Bewohner sogar sauberes Wasser kaufen. Das Problem ist überaus vielschichtig. Leider haben sich manche dieser negativen Verhaltensweisen zu einem Teil der Kultur entwickelt.

Für die Umsetzung des Projekts Very Very Important Fish brauchte ich die Unterstützung von Freiwilligen. Nachdem ich die Schauplätze kartografiert und eine Taktik entwickelt hatte, probte ich mit ihnen, Autos auf der Straße anzuhalten. Es ging vor allem darum, als konspirative Gruppe für rund zwei Stunden die Straße zu „hacken“. Für diese Aufgabe wählten wir diesmal gelbe Fahnen als unsere Waffen. In Jakarta ist diese Fahne allgemein als Symbol des Todes bekannt. In tropischen Regionen setzt die Verwesung einer Leiche sehr schnell ein, daher müssen die Toten sobald wie möglich beerdigt werden. Während der Hauptverkehrszeit ist es aber für eine größere Gruppe von Trauergästen sehr schwierig, in ihren Autos zum Friedhof zu gelangen, weswegen die gelbe Fahne so nützlich ist. Andere Fahrzeuge räumen einem Konvoi, der gelbe Fahnen mit sich führt, Vorfahrt ein. Dies ist ein ungeschriebenes Gesetz, gegen das selbst die Polizei nichts tun kann. Ich nutzte dieses System, um auf der Straße zwischen Jakarta und dem rund 30 Kilometer entfernten Depok Fahrzeuge anzuhalten. Ich verlieh dieser Aktion zusätzlich eine ökologische Botschaft, indem ich einen kleinen Fisch von einem stark verschmutzten in einen saubereren Fluss überführte.

Im Frühsommer 2011 fragte mich ein Freund in einem schummrigen Café in einem Vorort von Den Haag: „Für wen tust du das eigentlich?“ Für ein paar Schrecksekunden erstarrte mein Körper in einem eisigen Luftzug und meine Augen hörten auf zu blinzeln. Ich versuchte herauszufinden, was in meinem Kopf vor sich ging. Was habe ich im Sinn? Da die Macht in den Händen von Eliten liegt, die nie zufrieden sind, mit dem, was sie haben, ist es gar nicht so einfach herauszufinden, welcher Sache man sein Leben widmen soll. In der Vergangenheit waren die Menschen bereit, für ihre Nation und ihr Land zu sterben, doch heute ist Nationalismus nur noch ein Propagandawerkzeug, mit dem die Konzerne ihre Macht sichern. Die Religion, die über Jahrtausende hinweg die Menschen zu fast allem bewegen konnte, hat aufgrund eines rapiden kulturellen Wandels zugunsten des Ökonomischen an Bedeutung verloren. Ironischerweise warnen die 
Religionen immer wieder vor den Gefahren des Materialismus. Selbst die letzte Bastion, die Familie, verliert an Einfluss. Die Technik, die unser Leben angeblich einfacher machen soll, hat uns in Wahrheit zu isolierten Individuen gemacht und die Familien gespalten. Die Globalisierung scheint der Welt ein veritables Aussehen zu verleihen, aber die Welt macht dabei ein unglückliches Gesicht. Einige Sekunden lang war ich sprachlos und mein Herz schlug immer schneller. Dann sah ich Liveberichte im Fernsehen von den Bränden im Kernkraftwerk von Fukushima. Ich atmete tief durch und antwortete: „Um die Welt wieder zum Lächeln zu bringen!“

Aus dem Englischen übersetzt von Nikolaus Gramm.

\section{Endnoten}

[1] vgl. http://www.youtube.com/watch?v=u5y6ZNIrTHU)gl.

[2] Luchtalarm ist niederländisch und bedeutet Probealarm.

\section{Autor_innen}

Irwan Ahmett und Tita Salina (Mitautorin des Videos, siehe HTML) sind ein Künstler- und Designer-Duo. Sie leben in Jakarta. Sie kreieren Aktivitäten, die das Bewusstsein für die Wirklichkeit ihrer Stadt stimulieren und die Öffentlichkeit involvieren. Ihre Interventionen haben sie auch in anderen Teilen der Welt durchgeführt, unter anderem in Japan und verschiedenen europäischen Ländern.

irwanahmett@gmail.com

\section{Hacking Public Space and Influencing People}

In 1942, after three and a half centuries of colonization by the Dutch, Japan, as "the older Asian brother," promised to liberate Indonesia. Instead, the Japanese became the colonizers for the next three and a half years. When the atomic bombs fell on Hiroshima and Nagasaki and Japan lost the war, Indonesia was still a colony. Freedom fighters proclaimed the Republic of Indonesia on August 17th, 1945. But the British, US-American, and of course Dutch allied forces were not willing to see the colony go. One month after Soekarno and Hatta (Indonesia's first president and vice president, respectively) proclaimed independence, the Dutch returned and the allies refused to acknowledge Indonesia's independence. Unilaterally they flew the Dutch flag from high buildings. This provocative action escalated the situation until an incident at Yamato Hotel, Surabaya, East Java: the Indonesian people forced the Dutch to lower the tricolored (red, white, blue) flag, which culminated in a bloody battle. [...] One thing I take as a good note from that incident is the ripping of the flag at Yamato Hotel. The flagpole on which the tricolored Dutch flag hung was climbed by a group of brave young men armed with spoils-of-war weapons and sharpened bamboo sticks. The battle was unequal because the allies had more powerful armaments, but the real power lay with these young men, 
who were prepared to die. At the end, the blue color of the Dutch flag was ripped off, leaving only the two colors, red and white, of the Indonesian flag. The picture of that heroic action, which was disseminated by the media, inflamed resistance movements across the whole nation. For me, this incident is the first intervention in public space in Indonesia, and it remains the most important influence in every intervention project I have done. [...] 
